\title{
Bangla Optical Digits Recognition using Edge Detection Method
}

\author{
Md. MosarrafHossain \\ (Electrical and Electronic Engineering, Eastern University, Bangladesh)
}

\begin{abstract}
This paper is based on Bangla Optical Digit Recognition (ODR) by the Edge detection technique. In this method, Bangla digit image converted into gray-scale which distributed by an $M$ by $N$ array form. Here input data are considered off-line printed digit's image which collected from computer generated image, scanned documents or printed text. After addressing the gray-scale image against a variable in the form of an $M$ by $N$ array, where the value of array pointers are shown 255 for total white space, 0 (zero) for total dark space and value between 255 and 0 for mix of white and dark space of the image. At the next process, four edgestouch points as well as each touch point's ratio use as parameters to determine each Bangla digit uniquely.
\end{abstract}

Keywords-Edge, image, gray-scale, Matrix, $O D R$.

\section{Introduction}

Human beings are gifted with natural intelligence to recognize letters, voice, numbers, objects and any kind of optically recognizable characters. However, making a machine to solve these types of problems is a very difficult task. Pattern recognition is one of the important components of artificial intelligence. Interest in pattern recognition is aligned with the enormous amount of information that we encounter in our daily life. Consequently, computerization is desperately needed to handle this huge information. One of the difficult problems in the field of pattern recognition is Digits Recognition. Since the variation of the objects within each class is high, besides this, objects from different classes may be quite similar. Although there are challenges but the ideas and methodologies have been using to solve this problem would be very useful in many of the pattern recognition problems that include large volume of real-world data. In digits recognition task, formerly a digit is scanned, other preprocessing tasks need to pass before feature extraction and finally classification by a certain methodology.

Over the past three to four decades, many different methods have been explored and used in this field [1][2], including statistical, structural and syntactic methods, mathematical transforms, template (or model) matching, neural network and expert systems. In general, algorithms with good performance have either large descriptive complexity or computationally heavy to precisely identify individual character based on the database. However, more worksare still required before approaching to the human performance.

In this paper, I am going to discuss a first Bangla (Local language)DigitsRecognition system using detection of four (Top, bottom, right and left) edges touch points and its ratio. This systemworks Off-line. Detection of Edge Touch Points (ETP) algorithm is working on the specified information or parameters using logic instead of central database which makes the method faster than the conventional OCR system and economical. Therefore this paper has been evaluated the performance of Edge Touch Point (ETP) algorithm as well as ratio of Edge Touch Points to recognizing Bangla Digits correctly by the machine and its applications.

\section{Different Area Of Optical Digits Recognition}

Optical Digit Recognition deals with the problem of recognizing optically processed digits. Proposed Bangla ODR is performed off-line after the printing has been completed, as opposed to on-line recognition where the computer recognizes the characters or digits as they are drawn. Both printed and handwritten characters may be recognized, but the performance is directly dependents upon the quality of the inputs.In the figure-1, briefly illustrates the different area of the OCR/ODR system.

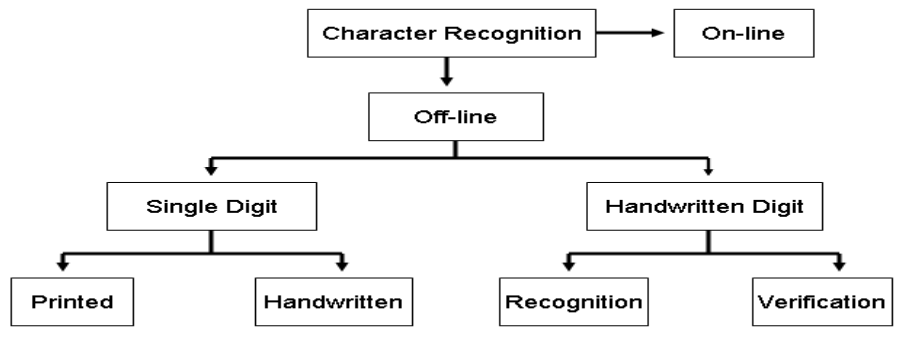

Fig. - 1: Different area of Optical Character/Digit Recognition 


\section{Components Of An OCR/ODR System}

A typical OCR/ODR system consists of several components. In figure- 2, a common setup [2] is illustrated. The first step in the process is to digitize the analog document using an optical scanner. When the regions containing texts are located, each symbol is extracted through a segmentation process. The extracted symbols may then be preprocessed, eliminating noise to facilitate the extraction of features in the next step and finally recognize the characters/digits through some post processing.

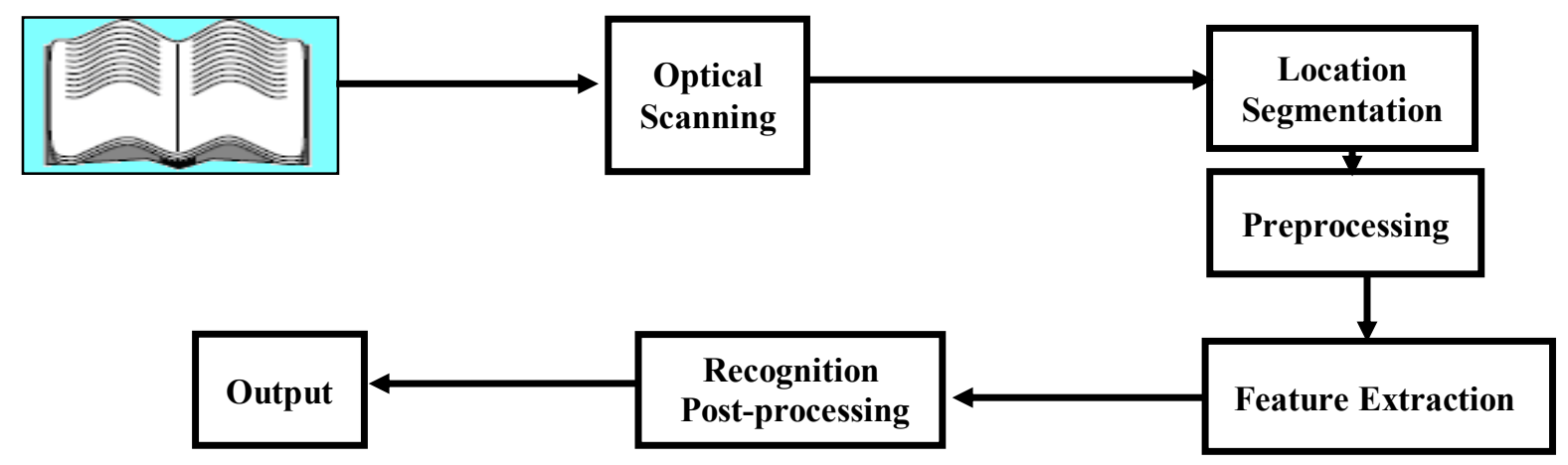

Fig.- 2: Components of typical OCR/ODR system

\section{Bangla Digits}

In the following table, given a complete list of Bangla digits corresponding to English digits those need to recognize by machine using the proposed method. Observing the following table, anyone can experienced that the shape of the Bangla digit of "o" (zero), "२" (two) and " 8 " (four) are almost similar with English digits "0" (zero), "2" (two) and "8" (eight) respectively.

TABLE- 1: Bangla digits corresponding to English digits

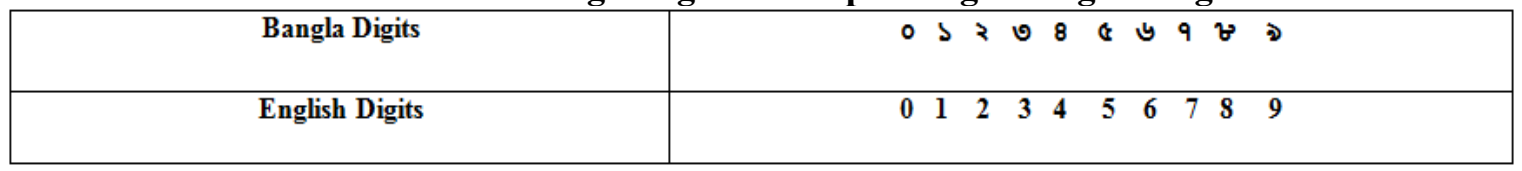

\section{Successful Works On OCR For Bangla Characters With Different Methods}

TABLE- 2: Most successful works done on OCR for Bangla characters.

\begin{tabular}{|c|c|c|}
\hline Name & Proposed Method & Accuracy \\
\hline $\begin{array}{c}\text { MuttakinurRahmanChowdhury (Shouro) [3] } \\
\text { [4] [12] }\end{array}$ & OCRopus & $98 \%$ \\
\hline Adnan Md.Shoeb [7] & $\begin{array}{c}\text { Kohonen Network } \\
\text { approach }\end{array}$ & $97.16 \%$ \\
\hline Bhattacharya \&Choudhuri [11] & Multi-resolution wavelet analysis and majority voting \\
\hline ASM MahabubMorshed et.al.[10] & Neural network for postal code recognition & $92.2 \%$ \\
\hline Dr. M AbulKashem [9] & Multilayer feed forward neural network & $97 \%$ \\
\hline ArijitSarkar [8] & Particle Swarm Optimization & $95.10 \%$ \\
\hline
\end{tabular}

\section{Proposed Method}

The proposed system works on the basis of Edge Touch Points (ETP) detection method and the ratio of the touch point which use to determine each Bangla digit uniquely for different fonts. The method of the Digit image recognition undergoes collecting and sorting out different fonts, re-shaping image, convertingRGB image into gray-scale, image processing, feature extraction, and classification. After feature extraction, each digit image is represented as a feature matrix, which is fed to a classifier for obtaining the class identity. The feature vectors of sample are used to learn the parameters of the classifier. Since Bangla digit provides gray-scale image, so proposed a process for recognition on gray-scale images directly to improve the recognition performance. The steps of the projected method are: 1) Flow chart.2) Image processing and 3) uniquely digit classification. All the mentioned steps implemented using the computer programming languageof MATLAB. 


\subsection{Flowchart}

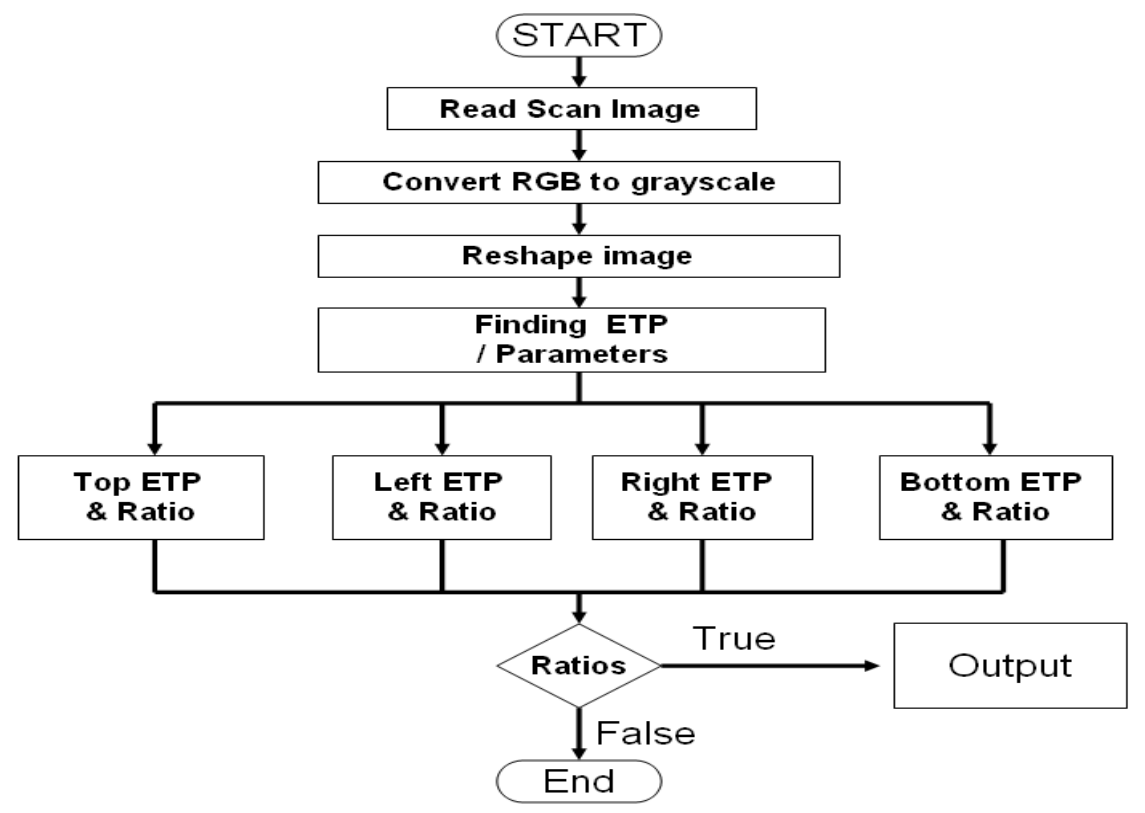

\subsection{Image processing}

Fig.-3: Flow chart

First of all, the raw input data that is printed bangle digit's image from optical scanningselected from widely used fonts which re-shaped and save in the same directory so that it can read easily in the MATLAB platform as of the following figure.

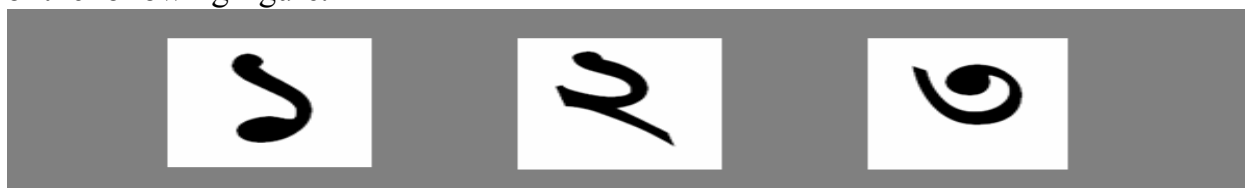

Fig.- 4: Scanned or printed Bangla digit images as input.

Then declare or save the image data against a variable (i.e. y) in MATLAB platform that can recall later like the following figure.

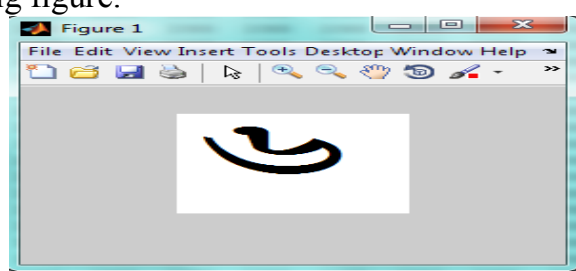

Fig. - 5: (Sutonny72emj6)

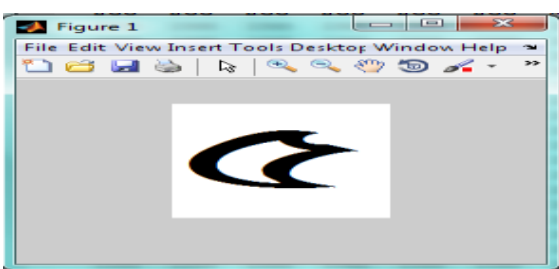

Fig. - 6: (Padma96emj5)

The return value of "y" is an array containing the image data. If the file contains a gray-scale image, $y$ is an M-by-N array. If the file contains a true color (RGB) image, y is an M-by-N-by-3 array. For TIFF files containing color images that use the CMYK color space, in this case,y is an M-by-N-by-4 array. For the file format [5], following are the companionable formats with our proposed method, listed in alphabetical order.

BMP- Windows Bitmap, CUR- Cursor File, GIF- Graphics Interchange Format, HDF4- Hierarchical Data Format, ICO- Icon File, JPEG- Joint Photographic Experts Group, JPEG 2000- Joint Photographic Experts Group 2000, PBM- Portable Bitmap, PCX- Windows Paintbrush, PGM- Portable Gray map, PNG- Portable Network Graphics, PPM- Portable Pixmap and RAS- Sun Raster.

In the proposed method, used PNG - Portable Network Graphics file format. Initially, whenever a true color digit images read or declare against any variable which is an $\mathrm{M}$ by $\mathrm{N}$ by 3 arrays. Each array pointer has a value as 255 for no data (total white), 0 for full of data (total black) and for mix of black and white data, the value of pointer will be any value between 255 and 0 (zero), which solely depend on the digit's image. For the sakeof analysis, converted true color image into gray-scale which expressed by an $\mathrm{M}$ by $\mathrm{N}$ arrays and this process substantially reduces unwanted data and noise. 

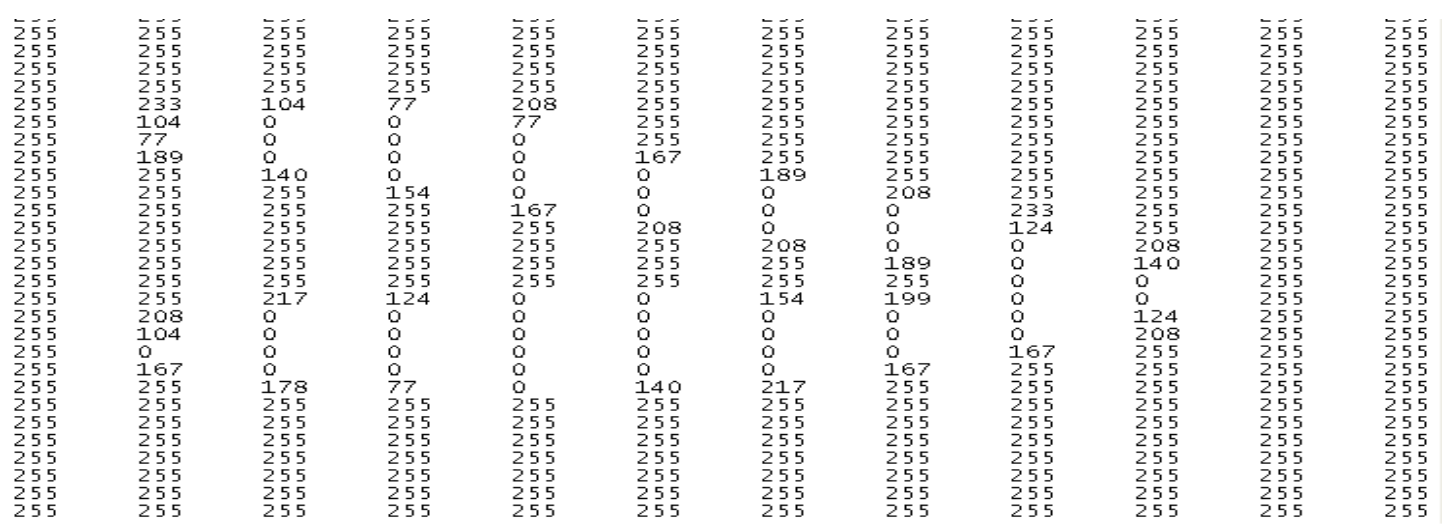

Fig.- 7: An $M$ by $N$ array for the image of Bangla digit one (১)

At this stage, performed elementary operation along rows and columns to omit 255 valuesand get required field of the dark area of the image. With the help of MATLAB, further re-shaped the image of Bangla digit as rectangular shape containing only the property of the image as follow
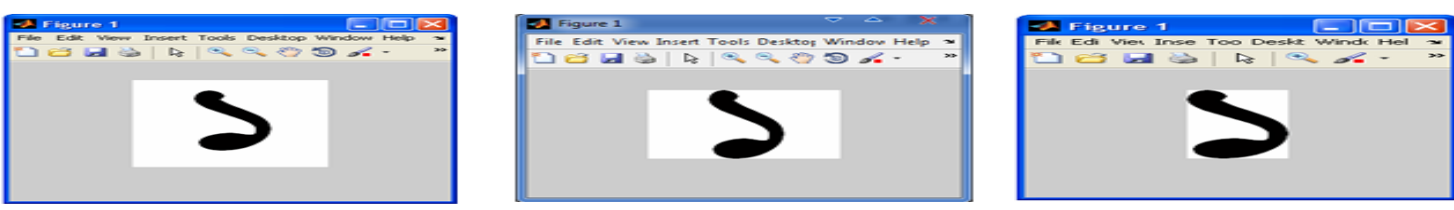

Fig. - 8:Initial image

Fig.- 9: After row operation Fig. 10: After column operation

$\begin{array}{llll}233 & 104 & 77 & 208 \\ 104 & 0 & 0 & 77 \\ 77 & 0 & 0 & 0 \\ 189 & 0 & 0 & 0 \\ 255 & 140 & 0 & 0 \\ 255 & 255 & 154 & 0 \\ 255 & 255 & 255 & 167 \\ 255 & 255 & 255 & 255 \\ 255 & 255 & 255 & 255 \\ 255 & 255 & 255 & 255 \\ 255 & 255 & 255 & 255 \\ 255 & 217 & 124 & 0 \\ 208 & 0 & 0 & 0 \\ 104 & 0 & 0 & 0 \\ 0 & 0 & 0 & 0 \\ 167 & 0 & 0 & 0 \\ 255 & 178 & 77 & 0\end{array}$
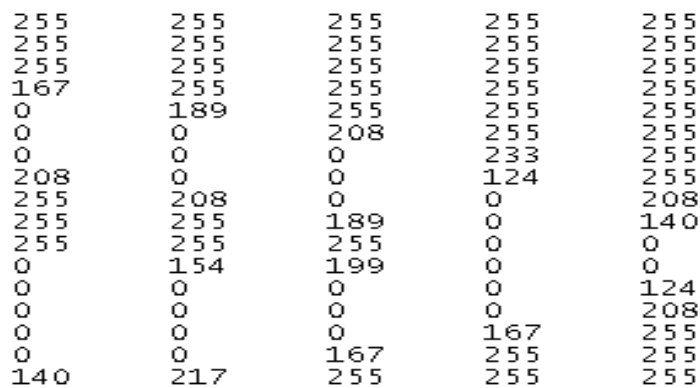

Fig.- 11:An $M$ by $N$ array for the image of Bangla digit one ( $\mathrm{S}$ ) after row and column operation.

\subsection{Uniquely digit classification}

Now it can easily determine the digit by Edge detection technique which constitutes using four touch points from the $\mathrm{M}$ by $\mathrm{N}$ image array. Touch points are top touch point, left touch point, right touch point and bottom touch point shown in the figure-12.

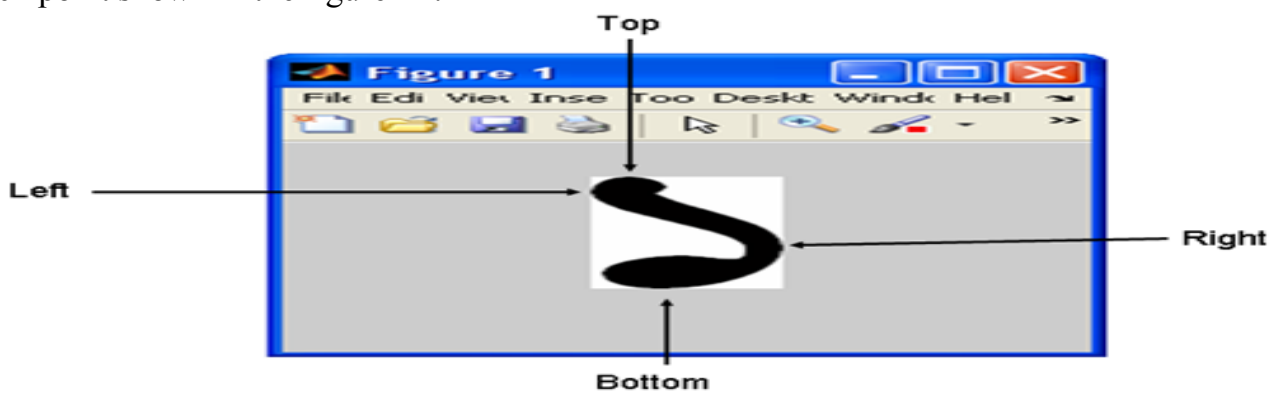

Fig.- 12: Location of touch points

After figured out the touch points of each edge of the image corresponding to the top, left, right and bottom sides of the image, calculated the position of the touch points along with the edges.

At this stage, found similar touch points for more than one digit when dealing with different fonts and this is a big challenge to identify each Bangla digit uniquely. To mitigate this problem, introduce a technique called ratio of touch points. For top touch point ratio, divide the number of top touch points by number of 
columns of the image matrix and similarly for the bottom touch point ratio but for left and right touch point ratio, divided by number of rows instead of columns of the image.

At this point, determined the range of the edge touch points and correspondingtouch point's ratio for each digit from the samples of 56 ( 7 fonts of each digit * 8 image sizes for each font) and run these parameters through"AND" and "OR" logical algorithm in the proposed method and successfully recognized Bangla digits according to the input raw images.

\section{Results}

In the proposed method, total 560 raw sample images of the 10 Bangla digits used ( 7 fonts for each digit $* 8$ different size of images for each font $* 10$ digits) as input and it can recognized 534 digits correctly. The accuracy for the proposed method is about $95 \%$. This method provides different accuracy for different fonts but only one font named "Parash", where it works with $100 \%$ accuracy because parameters for the Parash font falls middle of the range and did not fluctuate for different fonts and image size. A comparison of the accuracy for the different sample fonts present in the table- 3. On the other hand, for the some Bangla fonts where accuracy below $95 \%$ and the primary reasons are either the parameters aretoocloseor overlapped each other when increase the number of samples such as Dhakarchithi (93.75\%), Karnaphuli (93.75\%) and Sutonny $(92.50 \%)$. It is clear from the result of the below accuracy table for the different fonts that the percentage of accuracy closely related with variation of the shape of the Bangla digit images.

TABLE-3: Accuracy on different fonts of the Bangla digits.

\begin{tabular}{|c|c|c|c|c|c|c|c|c|c|c|c|}
\hline Fonts Name & & & $\mathbf{N}$ & $\mathbf{u}$ & $\mathbf{m}$ & b & e & $\mathbf{r}$ & $\mathrm{s}$ & & Accuracy \\
\hline Times New Roman (English) & $\mathbf{0}$ & 1 & 2 & 3 & 4 & 5 & 6 & 7 & 8 & 9 & N/A \\
\hline Parash & $\circ$ & $\mathrm{s}$ & 2 & 0 & 8 & ब & b & १ & b & ఉ & $100 \%$ \\
\hline BijoyChangma & $\circ$ & $s$ & २ & 0 & 8 & ब & b & १ & b & ه & $96.25 \%$ \\
\hline Chitra & o & $s$ & 2 & 0 & 8 & e & b & 9 & 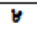 & D & $95 \%$ \\
\hline Dhakarchithi & 0 & s & p & 6 & 8 & a & b & 9 & 8 & $\$$ & $93.75 \%$ \\
\hline Dhanshirhi & 0 & d & \& & 0 & 8 & \& & a & १ & t & d & $96.25 \%$ \\
\hline Kamaphuli & 0 & ว & २ & 0 & 8 & 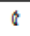 & ib & $q$ & $\mathrm{t}$ & के & $93.75 \%$ \\
\hline Sutonny & 0 & 2 & 2 & 0 & 8 & \& & g & 9 & $\mathrm{t}$ & 8 & $92.50 \%$ \\
\hline
\end{tabular}

\section{Applications}

During the last couple of decades, it has been seen that the widespread presence of commercial Optical Digit Recognition products meeting the requirement of different fields where mainly use English digits and now the proposed Bangla ODR method will open doors for our local language of Bangla digits. In Bangladesh,government owned banks have been using Bangla digits with unique font as of their Bank check number and maintain documents using Bangla digits. Those criteria makesthe proposed Bangla ODR technique suitable to atomization the banking operation of our government owned banks, reduce error, improve security features, increase customer satisfaction by providing efficient and faster services and reduce overall cost. Other prospective areas are automatic post code reading for mail sorting, Automatic vehicle number-plate reader, Automatic Text and data entry, Automatic Cartography and form readers and Automatic Vote counting machine where Bangla digits with specific fonts are used. Also this method can be applied for other languages as well.

\section{Conclusion}

Although, the proposed Bangla ODR method is not 100 percent successful for wide range of fonts but it works very fast compare to other existing ODR with 100\% accuracy for specific fonts such as Parash. Another feature is that the operation of the proposed Bangla ODR technique does not depend on database rather it dependents on the parameters of edge touch points and its ratio. As a result, this method is first, user friendly and economical to implement. In the future, the area of recognition of constrained print is expected to decrease. Importance will then be on the recognition of unconstrained writing, like omnifont [9] and handwriting. This is a challenge which requires improved recognition techniques. The potential of the future ODR algorithms seems to lie in the combination of different methods and the use of techniques that are able to utilize largercontext than current methodologies.

\section{Acknowledgements}

I would like to extend my sincere thanks to Mr. Abu Shafin Mohammad MahdeeJameel, Lecturer, department of EEE, Eastern University to give me the opportunity to work with an innovative topic as well as his valuable support to fulfill this research paper and honor his immense contributions throughout the process of study, constant encouragement and his direct guidance. His contribution in the preparation of the concept paper, literature, and writing this paper is highly acknowledged. 


\section{Reference}

[1] J-P. Caillot, Review of OCR Techniques.NR-note, BILD/08/087.

[2] J. Mantas, an Overview of Character Recognition Methodologies. PatternRecognition.

[3] http://code.google.com/p/ocropus-bengali, OCRopus Method, Retrieved on March 2011.

[4] http://www.bracuniversity.net/research/crblp/students.php, OCRopus and Tesseract method, Retrieved on February 2011.

[5] Help of MATLAB file format for image

[6] M. Bokser, Omnidocument Technologies, IEEE Proceedings, special issue on OCR.

[7] Adnan Md. ShoebShatil, A thesis paper on Bangla Optical character recognition using "Kohonen Network", CSE dept. Brac University.

[8] ArijitSarkar ,AurpanMajumder, Avijit Bose, “Ann in numerals Recognition \& Optimization using PSO”, CTCS-2010 at Assam University, 22-24 February 2010.

[9] Md. MahbubAlam and Dr. M. AbulKashem, A Complete Bangla OCR System for Printed Characters, ISSN 2078-5828 (print), volume 01, issue 01, Manuscript code: 100707.

[10] ASM MahabubMorshed, Automatic Sorting of mails by recognizing handwritten postal codes using neural network architectures.

[11] Chaudhuri BB, Pal U (1998), “A complete printed Bangla OCR system”, Pattern Recog., 31:531-549.

[12] MuttakinurRahmanChowdhury (Shouro), A thesis on Integration of Bangla script recognition support in OCRopus, CSE department, Brac University. 